\title{
On the Reliability of Acquiring Molecular Junction Parameters by Lorentzian Fitting of I/V curves
}

\author{
Vincent Delmas, Valentin Diez-Cabanes, Colin van Dyck, Elke Scheer, Karine Costuas, Jérôme \\ Cornil $^{*}$
}

\begin{abstract}
Fitting the I/V curves of molecular junctions by simple analytical models is often done to extract relevant molecular parameters such as energy level alignment or interfacial electronic coupling to build up useful property-relationships. However, such models can suffer from severe limitations and hence provide unreliable molecular parameters. This is illustrated here by extracting key molecular parameters by fitting computed voltage-dependent transmission spectra and by comparing them to the values obtained by fitting the calculated I/V curves with a typical Lorentzian model used in the literature. Doing so, we observe a large discrepancy between the two sets of values which warns us about the risks of using simple fitting expressions. Interestingly, we demonstrate that the quality of the fit can be improved by imposing the low bias conductance and Seebeck coefficient of the junction to be recovered in the fitting proce-

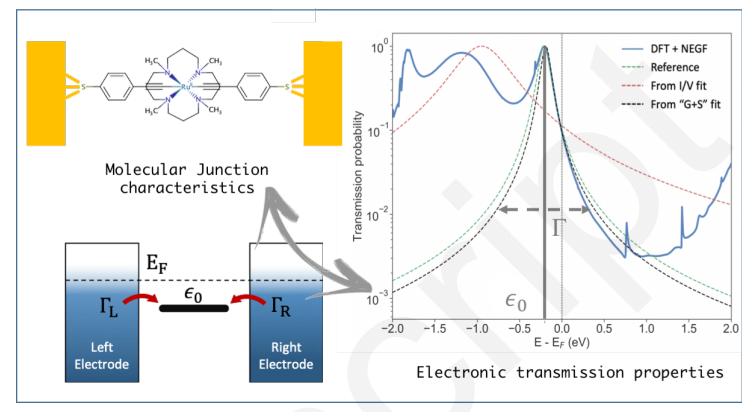
dure.
\end{abstract}

KEYWORDS: Molecular junctions, electronic transport, thermoelectricity, Fermi level alignment, organometallic compound, NEGF-DFT, computational chemistry

The field of molecular electronics aims at developing functional nano-electronic devices by inserting molecules between two metallic electrodes. The current-voltage (I/V) curves of such devices can be measured by several techniques such as conductive-AFM for self-assembled monolayers (SAMs) or Mechanically Controlled Break Junction (MCBJ) for single molecules. ${ }^{1-}$

${ }^{4}$ The electrical characteristics are governed by two key molecular parameters which are often voltage dependent: the energetic position of the molecular electronic levels responsible for charge transport with respect to the Fermi energy of the electrodes $\left(\varepsilon_{0}\right)$ which is not easy to measure ${ }^{5}$ and the electronic coupling strength between these orbitals and the electronic states of the electrodes $(\Gamma)$ that cannot be accessed directly experimentally. Although Ultraviolet/Inverse Photoelectron Spectroscopy (UPS/IPES) are typically used to infer the position of the electronic levels with respect to the Fermi level of a substrate in a SAM configuration, the relative position of the electronic levels is expected to be different in a molecular junction configuration (due to additional interfacial electronic coupling, built-in fields ${ }^{6}$ and image charge effects when a second electrode is introduced). ${ }^{7}$

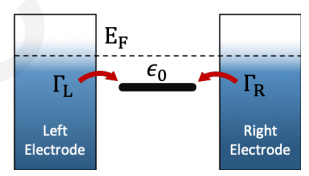

Figure 1. Simplified scheme featuring a single transporting molecular level of energy $\varepsilon_{0}$ (here the HOMO) coupled by a coupling strength $\Gamma_{\mathrm{R}}$ to the right electrode and $\Gamma_{\mathrm{L}}$ with the left electrode. The Fermi energy $\left(\mathrm{E}_{\mathrm{F}}\right)$ is represented by the dashed line.

At the present stage, $\varepsilon_{0}$ and $\Gamma$ parameters are mostly deduced by fitting experimental I/ $\mathrm{V}$ curves, considering that the electronic transmission at the origin of the current is of Breit-Wigner resonance type (Lorentzian-type shape); this model considers a single molecular level (generally the HOMO or LUMO of the molecule) at the energy $\varepsilon_{0}$ coupled to the electronic states of the electrodes (Figure 1). ${ }^{8-15}$ The full width at half maximum of this Lorentzian reflects the amplitude of the electronic coupling $\Gamma$. The Lorentzian-type expression of the electronic transmission $\tau(\mathrm{E})$ at a given energy $E$ is expressed as:

$$
\tau(E)=\frac{4 \Gamma_{L} \Gamma_{R}}{\left(E-\epsilon_{0}\right)^{2}+\left(\Gamma_{L}+\Gamma_{R}\right)^{2}}
$$

In the case of symmetric contacts between the molecule and the two electrodes, $\Gamma_{\mathrm{L}}$ and $\Gamma_{\mathrm{R}}$ are equal $\left(\Gamma_{\mathrm{L}}=\Gamma_{\mathrm{R}}=\Gamma\right)$ leading to the simplified expression:

$$
\tau(E)=\frac{4 \Gamma^{2}}{\left(E-\epsilon_{0}\right)^{2}+4 \Gamma^{2}}
$$

It is important to stress that this model suffers from severe limitations in considering that only one molecular transporting level is present close to the Fermi level, as evidenced theoretically by Neaton and co-workers. ${ }^{16}$ Using the DFT $+\Sigma$ (i.e., with self-energy corrections) formalism, some junctions show electronic transmissions which deviate importantly from a Lorentzian-shape curve. ${ }^{14}$ The single-level model is indeed expected to break down for molecules displaying several closelying energy levels in the transmission window. A second important hypothesis of this fitting model is to consider that the molecular parameters $\Gamma$ and $\varepsilon_{0}$ are bias voltage independent. In reality, and depending on how the applied voltage drops across the molecule, the level energy may be bias-dependent. Recent calculations clearly demonstrated that $\Gamma$ is a bias dependent parameter. ${ }^{17}$ Moreover, this model does not account for the typical decrease in the intensity of the transmission peak with increasing bias. Finally, if there are several molecules in the junctions, the total transmission is not simply the sum of the transmission 
spectrum of the individual molecules so that a prefactor accounting for cooperative effects should be introduced. Accordingly, we will only focus on single-molecule junctions in the following. Despite its significant restrictions, this model has been widely exploited by experimentalists to shed light on the electronic structure of molecular junctions and to establish relationships between the $\mathrm{I} / \mathrm{V}$ characteristics and the electronic structure of the molecular junction. In this context, our paper aims at addressing the reliability of this analytical procedure using quantum chemistry (QC) as a tool able to provide in parallel $\mathrm{I} / \mathrm{V}$ curves that can be fitted using the same models as previously described in literature and the actual calculated electronic parameters of a single-molecule junction based on calculated transmission functions. This allows us to evaluate the accuracy of the fitting models in recovering a correct amplitude for the two parameters using $\mathrm{I} / \mathrm{V}$ curves in comparison to the values calculated independently. It is important to emphasize at this stage that the scientific evidences given below are not dependent on the level of theory used. The present work highlights that much care has to be taken while using the single-level fitting procedure to extract the physical characteristics of a molecular junction.

In order to exemplify the problems that can arise from I/V curve fittings using this single-level model, we choose to evaluate the transmission properties of the trans-Ru(TMA $)\left(\mathrm{C} \equiv \mathrm{C}-\mathrm{C}_{6} \mathrm{H}_{4}-\mathrm{SH}\right)_{2}$ molecule (TMA = 1,5,9,13-tetramethyl-1,5,9,13-tetraazacyclohexadecane) grafted to gold electrodes. This system has been chosen as study case for several reasons. First, it is a symmetric molecular junction in which the metallic center $\mathrm{Ru}(\mathrm{II})$ is coordinated in a trans arrangement to conjugated (thiol)aryl-acetylide organic linkers. The rest of the central metal coordination sphere is completed by a tetra-chelating ligand via nitrogen atoms (Figure 2). This pseudo-octahedral environment stabilizes the metal center to fulfill the 18-electron rule conferring a high thermodynamic stability to the system. As lots of organometallic complexes, it displays a weak oxidation potential in comparison to most organic conjugated molecules, ${ }^{18}$ a feature which promotes molecular levels close to the Fermi energy of the electrodes. Since this system is known in its molecular form ${ }^{19,20}$ and closely-related molecular junctions have been successfully investigated experimentally by us and others ${ }^{21,22}$ it is highly suitable to experimental investigations. It has been chosen as study case among a large series of candidates that we have been screening since its calculated transmission properties show a high transmission peak close to the Fermi level with a quasiLorentzian shape, as required for this study.

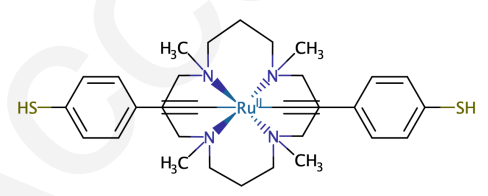

Figure 2. Chemical structure of trans- $\mathrm{Ru}(\mathrm{TMA})\left(\mathrm{C} \equiv \mathrm{C}-\mathrm{C}_{6} \mathrm{H}_{4}-\mathrm{SH}\right)_{2}$ (TMA = 1,5,9,13-tetramethyl-1,5,9,13-tetraazacyclo-hexadecane).

The molecular junction is built by grafting the molecule to two gold electrodes via sulfur-gold bonds on the hollow site of the gold (111) surface (thiol hydrogens removed, see Figure S1). The transmission spectra and I/V curves have been simulated in the coherent regime at the DFT+NEGF level using the ATK package software from Synopsys (see Supporting Information (SI) for additional computational details). ${ }^{23}$ The calculated transmission spectrum given in Figure $3 \mathrm{~b}$ at zero bias $(\Delta \mathrm{V}=0$
V) displays a Lorentzian peak centered at $\varepsilon_{0}=-0.214 \mathrm{eV}$ below the Fermi level. A very narrow peak (hence not contributing to the current) is also found at $\varepsilon_{1}=-0.4 \mathrm{eV}$ and is associated to other states involving the molecular backbone, (see Figure S2). ${ }^{24-26}$ Nonetheless, the main transmission peak can be accurately fitted by a single level as shown below.

The Landauer formula used to estimate the current is given in Eq. 3, where $\mu_{\mathrm{L}, \mathrm{R}}$ is the chemical potential of the left (L) and right $(\mathrm{R})$ electrode, $T$ the temperature, $k_{b}$ the Boltzmann constant, and $f_{\mathrm{L}, \mathrm{R}}$ is the Fermi-Dirac function:

$$
\begin{gathered}
I=\frac{2 e}{h} \int_{-\infty}^{+\infty} \tau(E, V)\left[f_{L}\left(E, \mu_{L}\right)-f_{R}\left(E, \mu_{R}\right)\right] d E ; \\
f_{L, R}=\frac{1}{\exp \left(\frac{E-\mu_{L, R}}{k_{b} T}\right)+1}
\end{gathered}
$$

In the following, we will also refer to the Seebeck coefficient $S$ and the conductance $G . S$ corresponds to the bias that can be generated by a difference of temperature between the two electrodes $(S=-\Delta \mathrm{V} / \Delta \mathrm{T})^{8,27-30} S$ has been measured for many molecular junctions. ${ }^{31,32}$ This parameter can be calculated using the transmission spectrum at zero bias (in the linear regime approximation) and is formally defined at zero current $I$. A simplification of the current equation (Eq. 3) can thus be done (see SI, Eqs. S1-4) leading to Eq. 4. In this expression, the $L_{\mathrm{n}}$ terms are incorporating integrals of the Fermi-Dirac function at equilibrium $\left(f_{\text {eq }}\right)($ Eq. 5, Eq. 6) which describes the distribution of electrons/holes over energy states in the electrodes. ${ }^{8,27-30}$

$$
\begin{gathered}
S=-\frac{1}{T} \frac{L_{1}}{L_{0}} \\
L_{n}=-\frac{2 e}{h} \int \tau(E, V)\left(E-\mu_{e q}\right)^{n} \frac{\partial f_{e q}}{\partial E} d E \\
f_{e q}=\frac{1}{\exp \left(\frac{E-\mu_{e q}}{k_{b} T}\right)+1}
\end{gathered}
$$

The conductance $G$ is calculated within the linear regime approximation as:

$$
G=L_{0} G_{0}
$$

with $G_{o}$ the quantum of conductance $(77.48 \mu \mathrm{S})$. The coupling $(\Gamma)$ and the energy $\left(\varepsilon_{0}\right)$ can be deduced in the single-level approximation directly from each calculated transmission spectrum by fitting the peak closest to the Fermi level by a BreitWigner function (Eq. 2). The implementation of the fitting procedures is detailed in SI. The obtained values can be considered as benchmark reference values for this study since they are directly issued from the DFT+NEGF transmission $(\tau)$ calculations (labelled later as $\Gamma_{\tau}$ and $\left.\varepsilon_{0-\tau}\right)$. One couple of values $\Gamma_{\tau}$ and $\varepsilon_{0-\tau}$ is thus obtained at each voltage (Table S1). The $\Gamma_{\tau}$ and $\varepsilon_{0-\tau}$ values given in Table 1 for several ranges of bias are the averaged values over the considered voltage range.

The calculated I/V curve of the $\mathrm{Au} \mid[$ trans-Ru(TMA) $(\mathrm{C} \equiv \mathrm{C}$ $\left.\left.\mathrm{C}_{6} \mathrm{H}_{4}-\mathrm{S}\right)_{2}\right] \mid \mathrm{Au}$ junction has been generated from calculated transmission spectra from 0 to $1 \mathrm{~V}$ by voltage steps of $0.1 \mathrm{~V}$. It is represented in Figure 3a. The physical characteristics of the molecular junction can be now deduced by a fitting procedure of the I/V curve, using the single-level model to obtain $\Gamma_{\mathrm{I} / \mathrm{V}}$ and 
$\varepsilon_{0-\mathrm{I} / \mathrm{V}}$ (Eq. 3). This was done using several voltage ranges in the I/V curve: (i) from 0 to $100 \mathrm{meV}$, a range of bias in which the current is proportional to the bias (linear regime); the transmission properties are slightly changed within this small potential window (no noticeable energy shift of $\varepsilon_{0}$ or $\Gamma$ ); (ii) in a larger voltage window ranging from 0 to $200 \mathrm{meV}$; (iii) for the total calculated I/V curve (from 0 to $1 \mathrm{~V}$ ). The results of these fits are given in Table 1 together with the reference values $\Gamma_{\tau}$ and $\varepsilon_{0-\tau}$ previously obtained. Although the three I/V curves fits are of very good accuracy $\left(\mathrm{R}^{2} \approx 1\right)$, the associated values $\varepsilon_{0-\mathrm{I} / \mathrm{V}}$ strongly deviate from $\varepsilon_{0-\tau}$, whatever the bias range considered. The $\Gamma_{\mathrm{I} / \mathrm{V}}$ values are at least doubled compared to $\Gamma_{\tau}$. As expected, the conductance $\mathrm{G}_{\mathrm{I} / \mathrm{V}}$ is close to the reference value since it is the fitted quantity. Interestingly, there is an order of magnitude difference between the Seebeck coefficient estimated from the different fitting ranges $\left(S_{I / V}\right)$ and the reference ones $\left(S_{\tau}\right)$ (see Table 1). The high quality of the I/V curve fits highly contrasts with the poor description of some of the molecular junction properties $\left(\varepsilon_{0}, \Gamma\right.$ and $\left.S\right)$. In the framework of the Transition Voltage Spectroscopy, $\varepsilon_{0}$ can also be estimated in principle as the minimum value in an $\mathrm{I} / \mathrm{V}$ versus $\ln \left(\mathrm{I} / \mathrm{V}^{2}\right)$ plot. ${ }^{11}$ This approach is not expected to provide reliable trends when the transmission peak is noticeably shifted with the bias voltage (Figure S3). However, in the case of weakly polarizable organic systems, such as oligophenylene derivatives (see Figure S4), the procedure based on the $\mathrm{I} / \mathrm{V}$ versus $\ln \left(\mathrm{I} / \mathrm{V}^{2}\right)$ plot to evaluate $\varepsilon_{0}$ is applicable. ${ }^{11,33}$

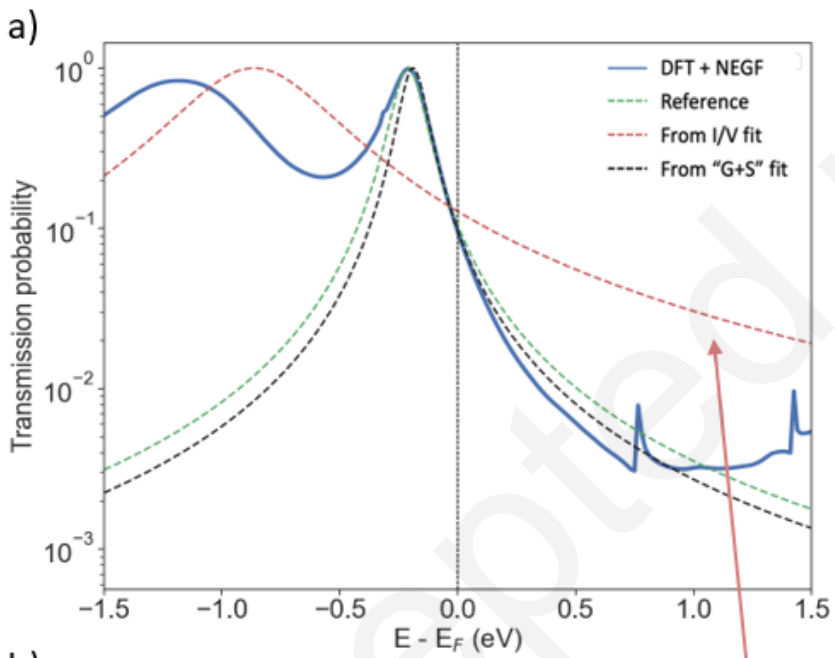

b)

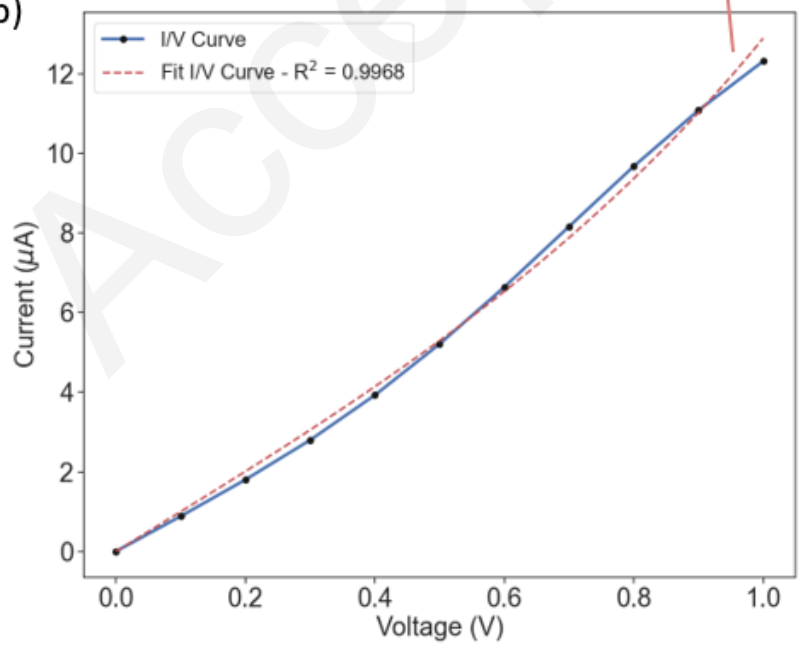

Figure 3. a) Calculated transmission spectrum at zero bias; Lorentzian curves generated from the transmission curve $(\tau)$, the I/V and the " $\mathrm{G}+\mathrm{S}$ " fits are given in dotted green, red and black lines respectively; see Figure S3 bias voltages up to $1 \mathrm{~V}$. b) I/V curve obtained from transmission calculations (steps of $0.1 \mathrm{~V}$ ) and fit of the $\mathrm{I} / \mathrm{V}$ curve.

Deviations are expected for a large integration voltage window due to the fact that $\epsilon_{0}$ and $\Gamma$ are bias dependent properties (Figure S5). The calculated $\varepsilon_{0-\tau}$ varies indeed linearly with the bias $\left(\epsilon_{0-\tau}(\mathrm{V})=-2 \cdot 0.10^{-4}-0.198 \mathrm{~V}\right.$ with $\left.\mathrm{R}^{2}=0.9914\right)$, and $\Gamma_{\tau}$ follows a $2^{\text {nd }}$ order polynomial progression with the voltage change $\left(\Gamma_{\tau}(V)=1.4 .10^{-8} V^{2}-2.9 .10^{-6} V+3.4 .10^{-2}\right.$ with $\left.\mathrm{R}^{2}=0.9968\right)$. Such evolutions revealed in this example are totally omitted in the single-model treatment. It surely induces a failure in obtaining the correct physical properties of a system when using a too large bias voltage window. On the other hand, if the fitting range is too small $\left(|\mathrm{eV}|<\epsilon_{0-\tau}\right)$, the I/V curves are mainly linear and no meaningful values of the two parameters can be inferred.

A major improvement can be obtained by imposing both the low bias conductance and the Seebeck coefficient of the junction to be recovered with a chosen accuracy (here above $96 \%$ ). The procedure adopted is explained in Figure 4 . This procedure is referred as the "G+S" method in Table 1. Doing so, the differences against the reference values of $\Gamma$ and $\varepsilon_{0}$ are strongly reduced, with the right order of magnitude recovered at low bias (Table 1, Figure 3a). This improvement can be rationalized by highlighting that in the I/V fit procedure, a perfect lorentziantype shape of the transmission is assumed. Thus, the more the system deviates from it, the more $\varepsilon_{0}$ and $\Gamma$ have probabilities to be erroneous even though $R^{2}$ is close to 1 (thus $G$ recovered). Imposing the constraint of also recovering $\mathrm{S}$ introduces a tolerance to the deviation from a perfect Lorentzian-shape that significantly improves the extracted parameters. Importantly, although the physical meaning of the parameters is improved, the corresponding I/V curve is apparently less satisfactory, with an $\mathrm{R}^{2}$ that significantly deviates from 1 . This relates to the fact that deviations are appearing when the linear regime approximation becomes irrelevant, i.e. at higher bias (see Figure S7). The "G+S" procedure can be applied to experimental I/V curves (see Table S3 for applications to data in Reference 34).

Table 1. $\Gamma(\mathrm{eV}), \varepsilon_{0}(\mathrm{eV}), S(\mu \mathrm{V} / \mathrm{K})$ and $G\left(\mathrm{G} / \mathrm{G}_{0}\right)$ obtained by a) application of the single-level method to fit $\tau(\mathrm{E})$ at each applied voltage (see Table S1); we report here the values averaged over the voltage range, $b$ ) fitting of the $\mathrm{I} / \mathrm{V}$ curve, $\mathrm{c}$ ) by adding $\mathrm{G}$ and $\mathrm{S}$ to the fitting procedure. The percentage errors are given in Table S2.

\begin{tabular}{l|l|r|r|r}
\multicolumn{2}{c|}{ Bias voltage range $(\mathrm{V}):$} & $0.0-0.1$ & $0.0-0.2$ & $0.0-1.0$ \\
\hline \multirow{2}{*}{ a) Fit of } & $\Gamma_{\tau}$ & 0.034 & 0.034 & 0.038 \\
$\tau(\mathrm{E})$ & $\epsilon_{0-\tau}$ & -0.214 & -0.223 & -0.311 \\
& $\mathrm{G}_{\tau} / \mathrm{G}_{0}$ & 0.114 & 0.115 & 0.136 \\
& $\mathrm{~S}_{\tau}$ & 88 & 86 & 67 \\
\hline \multirow{3}{*}{ b) Fit of I/V } & $\Gamma_{I / \mathrm{V}}$ & 0.169 & 0.105 & 0.165 \\
curve & $\epsilon_{0-I / V}$ & -0.947 & -0.590 & -0.863 \\
(Eq. 3) & $\mathrm{G}_{\mathrm{I} / \mathrm{V}} / \mathrm{G}_{0}$ & 0.114 & 0.114 & 0.129 \\
& $\mathrm{~S}_{\mathrm{I} / \mathrm{V}}$ & 14 & 23 & 15 \\
\hline \multirow{3}{*}{ c) Fit using } & $\Gamma_{" \mathrm{G}+\mathrm{S}^{\prime}}$ & 0.031 & $/$ & $/$ \\
the "G+S" & $\epsilon_{0-" G+S^{\prime \prime}}$ & -0.189 & $/$ & $/$ \\
procedure & $\mathrm{G}_{" \mathrm{G}+\mathrm{S}^{\prime \prime}} / \mathrm{G}_{0}$ & 0.113 & $/$ & $/$ \\
& $\mathrm{S}_{\mathrm{"}} \mathrm{G}+\mathrm{S}^{\prime \prime}$ & 88 & $/$ & $/$ \\
\hline
\end{tabular}




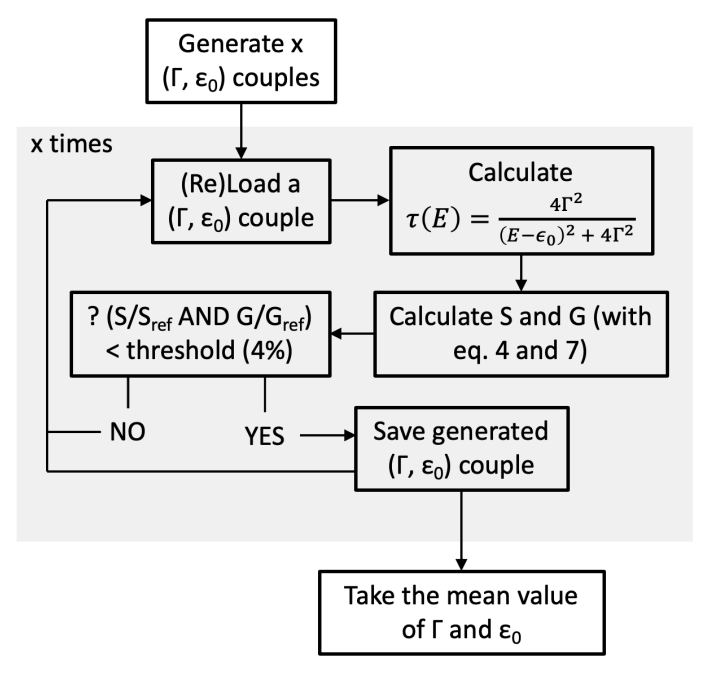

Figure 4. Workflow for the calculation of $G_{\tau} / G_{0}$ and $S_{\tau}$ which yields the $\left(\Gamma, \varepsilon_{0}\right)$ couple within the " $\mathrm{G}+\mathrm{S}$ " method. $\Gamma$ ranges from 0.01 to $0.1 \mathrm{eV}$ and $\varepsilon_{0}$ range from -2 to $0 \mathrm{eV}$.

Restricting the fitting to small voltage segments of the I/V curve could be seen as an alternative since the $\varepsilon_{0}$ and $\Gamma$ are not supposed to significantly change in each bias window. As shown in Figure S6, this procedure fails in reproducing the right bias dependence of the transmission spectra.

In conclusion, we have demonstrated that using a Breit-Wigner resonance type model to fit experimental or calculated I/V curves to get an insight into the electronic and transmission properties of molecular junctions can lead to significant errors in the evaluation of the molecular parameters $\varepsilon_{0}$ and $\Gamma$. We have shown that the correct position and width of the Lorentzian resonance cannot be reconstructed from the fitted values, even for a case study which presents a quasi-ideal transmission peak shape. The level of accuracy of the fitting procedure in the description of the physics has been strongly improved when considering simultaneously the conductance and the Seebeck coefficient of the junction that are both accessible experimental parameters.Erreur ! Le signet n'est pas défini. We hope that our analysis will drive further experimental efforts to improve the reliability of the traditionally done Lorentzian fitting procedures by measuring the Seebeck coefficient in combination with the conductance.

\section{ASSOCIATED CONTENT}

\section{Supporting Information}

The Supporting Information is available free of charge on the ACS Publications website.

\section{AUTHOR INFORMATION}

\section{Corresponding authors}

Karine Costuas - Univ Rennes, CNRS, ISCR (Institut des Sciences Chimiques de Rennes) - UMR 6226, F-35000 Rennes, France; (iD orcid.org/0000-0003-0338-0494;

Email: karine.costuas@univ-rennes1.fr

Jérôme Cornil - University of Mons, Laboratory for Chemistry of Novel Materials, Place du Parc 20, B-7000 Mons, Belgium; (i) orcid.org/0000-0002-5479-4227

Email: jerome.cornil@umons.ac.be

\section{Authors}

Vincent Delmas - Univ Rennes, CNRS, ISCR (Institut des Sciences Chimiques de Rennes) - UMR 6226, F-35000 Rennes, France;

Valentin Diez-Cabanes - Université de Lorraine, Laboratoire de Physique et Chimie Théoriques, CNRS, F-54506 Vandoeuvre-lesNancy, France; (D) orcid.org/0000-0002-6234-2749

Colin van Dyck - University of Mons, Department of Physics, Place du Parc 20, B-7000 Mons, Belgium; (D orcid.org/0000-00032853-3821

Elke Scheer - University of Konstanz, Department of Physics, 78457 Konstanz, Germany; (D) orcid.org/0000-0003-3788-6979

\section{Author Contributions}

The manuscript was written through contributions of all authors. All authors have given approval to the final version of the manuscript.

Notes

The authors declare no competing financial interest.

\section{ACKNOWLEDGMENTS}

V.D. acknowledges "Rennes Métropole" for financial support of his scientific stay in Mons. K.C. and V.D. Acknowledge support by the "Grand équipement national de calcul intensif (GENCI)" through HPC resources of CINES and IDRIS (grants 2019/2020A0080800649/A0060800649). The work in Mons is supported by the Belgian National Fund for scientific research (FR-FNRS). Computational resources have been provided by the Consortium des Équipements de Calcul Intensif (CÉCI), funded by the Fonds de la Recherche Scientifique de Belgique (F.R.S.-FNRS) under Grant $\mathrm{N}^{\circ} .2 .5020 .11$ and by the Walloon Region. J.C. is an FNRS research director. The French Embassy in Berlin is acknowledged for its financial support to the organization of scientific meetings between the authors (Procope structurant 2018).

\section{ABBREVIATIONS}

TMA, 1,5,9,13-tetramethyl-1,5,9,13-tetraazacyclohexadecane; PF, Power Factor; DFT, Density Functional Theory;

\section{REFERENCES}

(1) Wold, D. J.; Frisbie, C. D. Fabrication and Characterization of Metal-Molecule-Metal Junctions by Conducting Probe Atomic Force Microscopy. J. Am. Chem. Soc. 2001, 123 (23), 5549-5556.

(2) STM and AFM Studies on (Bio)Molecular Systems: Unravelling the Nanoworld; Samorì, P., Ed.; Topics in Current Chemistry; Springer Berlin Heidelberg: Berlin, Heidelberg, 2008; Vol. 285.

(3) Hong, W.; Valkenier, H.; Mészáros, G.; Manrique, D. Z.; Mishchenko, A.; Putz, A.; García, P. M.; Lambert, C. J.; Hummelen, J. C.; Wandlowski, T. An MCBJ Case Study: The Influence of $\pi$-Conjugation on the Single-Molecule Conductance at a Solid/Liquid Interface. Beilstein J. Nanotechnol. 2011, 2, 699-713.

(4) Wang, L.; Wang, L.; Zhang, L.; Xiang, D. Advance of Mechanically Controllable Break Junction for Molecular Electronics. Top Curr Chem (Z) 2017, 375 (3), 61.

(5) Fereiro, J. A.; McCreery, R. L.; Bergren, A. J. Direct Optical Determination of Interfacial Transport Barriers in Molecular Tunnel Junctions. J. Am. Chem. Soc. 2013, 135 (26), 9584-9587.

(6) Van Dyck, C.; Bergren, A. J. Large Built-In Fields Control the Electronic Properties of Nanoscale Molecular Devices with Dipolar Structures. Adv. Electron. Mater. 2018, 4 (5), 1700656.

(7) Diez-Cabanes, V.; Gonzalez, S. R.; Osella, S.; Cornil, D.; Van Dyck, C.; Cornil, J. Energy Level Alignment at Interfaces Between Au (111) and Thiolated Oligophenylenes of Increasing Chain Size: Theoretical Evidence of Pinning Effects. Advanced Theory and Simulations 2018, 1 (3), 1700020. 
(8) Huisman, E. H.; Guédon, C. M.; van Wees, B. J.; van der Molen, S. J. Interpretation of Transition Voltage Spectroscopy. Nano Lett. 2009, 9 (11), 3909-3913.

(9) Bâldea, I. Revealing Molecular Orbital Gating by Transition Voltage Spectroscopy. Chemical Physics 2010, 377 (1-3), 15-20.

(10) Cuevas, J. C.; Scheer, E. Molecular Electronics: An Introduction to Theory and Experiment; World Scientific series in nanoscience and nanotechnology; World Scientific: Singapore; Hackensack, NJ, 2010.

(11) Bâldea, I. Ambipolar Transition Voltage Spectroscopy: Analytical Results and Experimental Agreement. Phys. Rev. B 2012, 85 (3), 035442 .

(12) Briechle, B. M.; Kim, Y.; Ehrenreich, P.; Erbe, A.; Sysoiev, D.; Huhn, T.; Groth, U.; Scheer, E. Current-Voltage Characteristics of Single-Molecule Diarylethene Junctions Measured with Adjustable Gold Electrodes in Solution. Beilstein J. Nanotechnol. 2012, 3, 798-808.

(13) Xie, Z.; Bâldea, I.; Smith, C. E.; Wu, Y.; Frisbie, C. D. Experimental and Theoretical Analysis of Nanotransport in Oligophenylene Dithiol Junctions as a Function of Molecular Length and Contact Work Function. ACS Nano 2015, 9 (8), 8022-8036.

(14) Isshiki, Y.; Matsuzawa, Y.; Fujii, S.; Kiguchi, M. Investigation on Single-Molecule Junctions Based on Current-Voltage Characteristics. Micromachines 2018, 9 (2), 67.

(15) Xie, Z.; Bâldea, I.; Frisbie, C. D. Determination of Energy-Level Alignment in Molecular Tunnel Junctions by Transport and Spectroscopy: Self-Consistency for the Case of Oligophenylene Thiols and Dithiols on Ag, Au, and Pt Electrodes. Journal of the American Chemical Society 2019, 141 (8), 3670-3681.

(16) Quek, S. Y.; Choi, H. J.; Louie, S. G.; Neaton, J. B. Thermopower of Amine-Gold-Linked Aromatic Molecular Junctions from First Principles. ACS Nano 2011, 5 (1), 551-557.

(17) Liu, Z.-F.; Neaton, J. B. Voltage Dependence of Molecule-Electrode Coupling in Biased Molecular Junctions. J. Phys. Chem. C 2017, 121(39), 21136-21144

(18) Costuas, K.; Rigaut, S. Polynuclear carbon-rich organometallic complexes: clarification of the role of the bridging ligand in the redox properties. Dalton Trans. 2011, 40(21), 5643-5658.

(19) Wong, C.-Y.; Lee, F.-W.; Che, C.-M.; Cheng, Y. F.; Phillips, D. L.; Zhu, N. Spectroscopic Studies and Structures of Trans -Ruthenium(II) and Ruthenium(III) Bis(Cyanide) Complexes Supported by a Tetradentate Macrocyclic Tertiary Amine Ligand. Inorganic Chemistry 2008, 47 (22), 10308-10316.

(20) Doro, F. G.; Ferreira, K. Q.; da Rocha, Z. N.; Caramori, G. F.; Gomes, A. J.; Tfouni, E. The Versatile Ruthenium(II/III) Tetraazamacrocycle Complexes and Their Nitrosyl Derivatives. Coordination Chemistry Reviews 2016, 306, 652-677.

(21) Meng, F.; Hervault, Y.-M.; Norel, L.; Costuas, K.; Van Dyck, C.; Geskin, V.; Cornil, J.; Hng, H. H.; Rigaut, S.; Chen, X. Photo-Modulable Molecular Transport Junctions Based on Organometallic Molecular Wires. Chem. Sci. 2012, 3, 3113-3118.

(22) Milan, D. C.; Vezzoli, A.; Planje, I. J.; Low, P. J. Metal Bis(Acetylide) Complex Molecular Wires: Concepts and Design Strategies. Dalton Trans. 2018, 47, 14125-14138.

(23) QuantumATK Q-2019.12 Synopsys (https://www.synopsys.com/silicon/quantumatk.html).

(24) Porod, W.; Shao, Z.; Lent, C. S. Resonance-Antiresonance Line Shape for Transmission in Quantum Waveguides with Resonantly Coupled Cavities. Phys. Rev. B 1993, 48 (11), 8495-8498.

(25) Göres, J.; Goldhaber-Gordon, D.; Heemeyer, S.; Kastner, M. A.; Shtrikman, H.; Mahalu, D.; Meirav, U. Fano Resonances in Electronic Transport through a Single-Electron Transistor. Physical Review B 2000, 62 (3), 2188-2194.

(26) Lambert, C. J. Basic Concepts of Quantum Interference and Electron Transport in Single-Molecule Electronics. Chemical Society Reviews 2015, 44 (4), 875-888.

(27) Paulsson, M.; Datta, S. Thermoelectric Effect in Molecular Electronics. Physical Review B 2003, 67 (24).

(28) Reddy, P.; Jang, S.-Y.; Segalman, R. A.; Majumdar, A. Thermoelectricity in Molecular Junctions. Science 2007, 315, 5.

(29) Zimbovskaya, N. A. Transport Properties of Molecular Junctions; Springer tracts in modern physics; Springer: New York, 2013.
(30) Zimbovskaya, N. A. Seebeck Effect in Molecular Junctions. Journal of Physics: Condensed Matter 2016, 28 (18), 183002.

(31) Widawsky, J. R.; Darancet, P.; Neaton, J. B.; Venkataraman, L. Simultaneous Determination of Conductance and Thermopower of Single Molecule Junctions. Nano Letters 2012, 12 (1), 354-358.

(32) Cui, L.; Miao, R.; Wang, K.; Thompson, D.; Zotti, L. A.; Cuevas, J. C.; Meyhofer, E.; Reddy, P. Peltier Cooling in Molecular Junctions. Nature Nanotechnology 2018, 13 (2), 122-127.

(33) Paulsson, M.; Datta, S. Thermoelectric Effect in Molecular Electronics. Physical Review B 2003, 67 (24).

(34) Reddy, P.; Jang, S.-Y.; Segalman, R. A.; Majumdar, A. Thermoelectricity in Molecular Junctions. Science 2007, 315, 5.

(35) Zimbovskaya, N. A. Transport Properties of Molecular Junctions; Springer tracts in modern physics; Springer: New York, 2013.

(36) Zimbovskaya, N. A. Seebeck Effect in Molecular Junctions. Journal of Physics: Condensed Matter 2016, 28 (18), 183002.

(37) Rodriguez-Gonzalez, S. Xie, Z. Galangau, O. Selvanathan, P. Norel, L.; van Dyck, C.; Costuas, K.; Frisbie, C. D.; Rigaut, S.; Cornil J. HOMO Level Pinning in Molecular Junctions: Joint Theoretical and Experimental Evidence. J. Phys. Chem. Lett. 2018, 9, 9, 2394-2403

(38) Cui, L.; Hur, S.; Akbar, Z. A.; Klöckner, J. C.; Jeong, W.; Pauly, F.; Jang, S.-Y.; Reddy, P.; Meyhofer, E. Thermal Conductance of Single-Molecule Junctions. Nature 2019, 572 (7771), 628-633. 